\title{
The Lower and Upper Forcing Edge-to-vertex Geodetic Numbers of a Graph
}

\author{
S.Sujitha \\ Department of Mathematics \\ Holy Cross College (Autonomous) \\ Nagercoil, India.
}

\begin{abstract}
A$ subset $T \subseteq S$ is called a forcing subset for $S$ if $S$ is the unique minimum edge-to-vertex geodetic set containing $T$. A forcing subset for $S$ of minimum cardinality is a minimum forcing subset of $S$. The forcing edge-to-vertex geodetic number of $S$, denoted by $f_{e v}(S)$, is the cardinality of a minimum forcing subset of $S$. The lower forcing edge-to-vertex geodetic number of $G$, denoted by $f_{e v}(G)$, is $f_{e v}(G)=\min \left\{f_{e v}(S)\right\}$, where the minimum is taken over all minimum edge-to-vertex geodetic sets $S$ in $G$. The upper forcing edge-to-vertex geodetic number of $G$, denoted by $f^{+}{ }_{e v}(G)$, is $f^{+}{ }_{e v}(G)=\max \left\{f_{e v}(S)\right\}$, where the maximum is taken over all minimum edge-to-vertex geodetic sets $S$ in $G$. These concepts were studied in [3], [4] and [9]. In this paper, we extend the study of lower and upper forcing edge-to-vertex geodetic numbers of graphs whose minimum edge-to-vertex geodetic sets containing antipodal edges.
\end{abstract}

Keywords: edge-to-vertex geodetic number, lower forcing edge-to-vertex geodetic number, upper forcing edge-to-vertex geodetic number.

AMS Subject Classification: 05C12.

\section{INTRODUCTION}

By a graph $G=(V, E)$, we mean a finite undirected connected graph without loops or multiple edges. The order and size of $G$ are denoted by $p$ and $q$ respectively. For basic definitions and terminologies we refer to [1]. For vertices $u$ and $v$ in a connected graph $G$, the distance $d(u, v)$ is the length of a shortest $u-v$ path in $G$. A $u-v$ path of length $d(u, v)$ is called a $u-v$ geodesic. A vertex $x$ is said to lie on a $u-v$ geodesic if $x$ is a vertex of a $u-v$ geodesic.

A geodetic set of $G$ is a set $S \subseteq V(G)$ such that every vertex of $G$ is contained in a geodesic joining a pair of vertices of $S$. The geodetic number $g(G)$ of $G$ is the minimum cardinality of a geodetic set and any geodetic set of cardinality $g(G)$ is called a geodetic basis or simply a $g$-set of $G$. A set $S \subseteq E(G)$ is called an edge-to-vertex geodetic set if every vertex of $G$ is either incident with an edge of $S$ or lies on a geodesic joining a pair of edges of $S$. The edge-to-vertex geodetic number $g_{e v}(G)$ of $G$ is the minimum cardinality of its edge-to-vertex geodetic sets and any edge-to-vertex geodetic set of cardinality $g_{e v}(G)$ is called an edge-to-vertex geodetic basis of $G$ or a $g_{e v}(G)$-set of $G$. This concept is studied in [8].

For any edge $e$ of a connected graph $G$, the edge-to-edge eccentricity $e_{3}(e)$ of $e$ is $e_{3}(e)=\max \{d(e, f): f$ $\in E(G)\}$. The minimum eccentricity among the edges of $G$ is the edge-to-edge radius, rad $G$ and the maximum eccentricity among the edges of $G$ is the edge-to-edge diameter, diam $G$ of $G$. Two edges $e$ and $f$ are antipodal if $d(e, f)=\operatorname{diam} G$ or $d(G)$. This concept was studied in [7]. A vertex $v$ is an extreme vertex of a graph $G$ if the subgraph induced by its neighbors is complete. An edge $e$ of a graph $G$ is called an extreme edge of $G$ if one of its end is an extreme vertex of $G$.

A wheel graph is a graph formed by connecting a single vertex to all vertices of a cycle and it is denoted by $W_{p}$. In this paper, $W_{p}$ denotes a wheel graph with $p+1$ vertices $\quad(p \geq 3)$ which is formed by connecting a single vertex to all vertices of a cycle of length $p$. The wheel graph has diameter two if $p>3$ and one if $p=3$. The graph $C_{4} \times K_{2}$ is often denoted by $Q_{3}$ and is called 3-cube. The triangular snake $T S_{n}$ is obtained from the path $P_{n}$ by replacing every edge of a path by a triangle $C_{3}$. The square 
of graph $G$ denoted by $G^{2}$ is defined to be the graph with the same vertex set as $G$ and in which two vertices $u$ and $v$ are joined by an edge in $G \Leftrightarrow 1 \leq d(u, v) \leq 2$. These concept were studied in [2],[5] and [6].

A subset $T \subseteq S$ is called a forcing subset for $S$ if $S$ is the unique minimum edge-to-vertex geodetic set containing $T$. A forcing subset for $S$ of minimum cardinality is a minimum forcing subset of $S$. The forcing edge-to-vertex geodetic number of $S$, denoted by $f_{e v}(S)$, is the cardinality of a minimum forcing subset of $S$. The lower forcing edge-to-vertex geodetic number of $G$, denoted by $f_{e v}(G)$, is $f_{e v}(G)=\min \left\{f_{e v}(S)\right\}$, where the minimum is taken over all minimum edge-to-vertex geodetic sets $S$ in $G$. The upper forcing edge-to-vertex geodetic number of $G$, denoted by $f{ }_{e v}^{+}(G)$, is $f^{+}{ }_{e v}(G)=$ $\max \left\{f_{e v}(S)\right\}$, where the maximum is taken over all minimum edge-to-vertex geodetic sets $S$ in $G$. These concepts were studied in [3],[4] and [9]. In this paper we study some more properties about the lower and upper forcing edge-to-vertex geodetic numbers in minimum edge-to-vertex geodetic sets of connected graphs.

Throughout the following $G$ denotes a connected graph with at least three vertices. The following Theorems are used in the sequel.

Theorem 1.1.[9] For every connected graph $G, 0 \leq f^{+}{ }_{e v}(G) \leq g_{e v}(G)$.

Theorem 1.2. [3] For every connected graph $G, 0 \leq f_{e v}(G) \leq g_{e v}(G)$.

Theorem 1.3. [3] Let $G$ be a connected graph. Then

a) $f_{e v}(G)=0$ if and only if $G$ has a unique minimum edge-to-vertex geodetic set.

b) $f_{e v}(G)=1$ if and only if $G$ has at least two minimum edge-to-vertex geodetic sets, one of which is a unique minimum edge-to-vertex geodetic set containing one of its elements, and

c) $f_{e v}(G)=g_{e v}(G)$ if and only if no minimum edge-to-vertex geodetic set of $G$ is the unique minimum edge-to-vertex geodetic set containing any of its proper subsets.

Theorem 1.4. [9] Let $G$ be a connected graph. Then

a) $f^{+}{ }_{e v}(G)=0$ if and only if $G$ has a unique minimum edge-to-vertex geodetic set.

b) $f_{e v}^{+}(G)=1$ if and only if $G$ has at least two minimum edge-to-vertex geodetic sets, in which one element of each minimum edge-to-vertex geodetic set of $G$ does not belong to any other minimum edge-to-vertex geodetic set of $G$.

c) $f^{+}{ }_{e v}(G)=g_{e v}(G)$ if and only if there exists at least one minimum edge-to-vertex geodetic set of $G$ which does not contain any proper forcing subsets.

\section{The Lower and UPPer Forcing Edge-to-Vertex GeOdetic Numbers of A GRAPH}

The following Lemma gives the bound for the lower and upper forcing edge-to-vertex geodetic numbers of a graph. It is the extension of the results proved in [3] and [9].

Lemma 2.1. For every connected graph $G, 0 \leq f_{e v}(G) \leq f^{+}{ }_{e v}(G) \leq g_{e v}(G)$.

Proof. In [3] and [9], we proved the results $0 \leq f_{e v}(G) \leq g_{e v}(G)$ and $0 \leq f_{e v}^{+}(G) \leq g_{e v}(G)$. Therefore, to conclude the lemma we need to prove $f_{e v}(G) \leq f^{+}{ }_{e v}(G)$. By the definition of the lower and upper forcing edge-to-vertex geodetic number, we see that $f_{e v}^{+}(G)$ is the maximum of all forcing edge-tovertex geodetic numbers of the minimum edge-to-vertex geodetic sets, and $f_{e v}(G)$ is the minimum of all forcing edge-to-vertex geodetic numbers of the minimum edge-to-vertex geodetic sets. Hence the inequality is obvious.

Example 2.2. The bounds in Lemma 2.1 are sharp. Consider the non-trivial tree $G=T$. Since the tree has a unique minimum edge-to-vertex geodetic set, and by Theorem 1.4(a) and Theorem 1.3(a), $f$ ${ }_{e v}(G)=0, f_{e v}(G)=0$ so that $0=f_{e v}(G)=f^{+}{ }_{e v}(G)$. Also, in [3] and [9], for an even cycle $C_{2 p}(p=$ $2,3 \ldots), f^{+}{ }_{e v}(G)=1=f_{e v}(G)$ and $g_{e v}(G)=2$. Moreover, all the inequalities in Lemma 2.1 are strict. 
For the wheel graph $G=W_{l 0}, f^{+}{ }_{e v}(G)=3, f_{e v}(G)=2$ and $g_{e v}(G)=4$. Hence $0<f_{e v}(G)<f^{+}{ }_{e v}(G)<$ $g_{e v}(G)$.

In [3] and [9], we showed the result, $f^{+}{ }_{e v}(G)=0$ if and only if $G$ has a unique minimum edge-tovertex set and also, $f_{e v}(G)=0$ if and only if $G$ has a unique minimum edge-to-vertex set. The following lemma is an extension of that result.

Lemma 2.3. For a connected graph $G, f_{e v}(G)=0$ if and only if $f^{+}{ }_{e v}(G)=0$.

Proof. The proof is obvious.

Theorem 2.4. For a 3-cube graph $G=Q_{3}$, a set $S \subseteq E(G)$ is a minimum edge-to-vertex geodetic set if and only if $S$ consists of a pair of antipodal edges.

Proof. Let the vertices of $Q_{3}$ be $v_{1}, v_{2}, v_{3}, \ldots, v_{8}$. Without loss of generality, we take $v_{1}, v_{2}, v_{3}$ and $v_{4}$ are the vertices of the outer square and $v_{5}, v_{6}, v_{7}$ and $v_{8}$ are the vertices of the inner square of the 3cube. Then the edges $v_{1} v_{5}$ and $v_{3} v_{7}$ are a pair of antipodal edges. Let $S=\left\{v_{1} v_{5}, v_{3} v_{7}\right\}$. Clearly, $S$ is a minimum edge-to-vertex geodetic set of $Q_{3}$. Conversely, let $S$ be a minimum edge-to-vertex geodetic set of $Q_{3}$. Then $g_{e v}\left(Q_{3}\right)=|S|$. Let $S^{\prime}$ be any set of pair of antipodal edges of $Q_{3}$. Then as in the first part of this theorem, $S^{\prime}$ is a minimum edge-to-vertex geodetic set of $Q_{3}$. Hence $\left|S^{\prime}\right|=|S|$. Thus $S=$ $\{u v, x y\}$. If $u v$ and $x y$ are not antipodal, then any vertex that is not on the $u v-x y$ geodesic does not lie on the $u v-x y$ geodesic. Thus $S$ is not a minimum edge-to-vertex geodetic set, which is a contradiction.

Theorem 2.5. For a 3-cube graph $G=Q_{3}, f_{e v}(G)=f^{+}{ }_{e v}(G)=1$.

Proof. By Theorem 2.4, every minimum edge-to-vertex geodetic set of $Q_{3}$ consists of pair of antipodal edges. Hence $Q_{3}$ has two independent minimum edge-to-vertex geodetic sets and it is clear that each singleton set is the minimum forcing subset for exactly one minimum edge-to-vertex geodetic set of $Q_{3}$. Hence it follows from Theorem 1.3 (a) and (b) that $f_{e v}\left(Q_{3}\right)=1$. Also, from Theorem 1.4 (a) and (b) that $f^{+}{ }_{e v}\left(Q_{3}\right)=1$. Thus $f_{e v}(G)=f^{+}{ }_{e v}(G)=1$.

Theorem 2.6. Let $G$ be a connected graph with at least two $g_{e v}$-sets. If every minimum edge-tovertex geodetic sets of $G$ containing antipodal edges, then $f_{e v}(G)=1=f^{+}{ }_{e v}(G)$.Proof. Let $G$ be a connected graph. Suppose $S_{i}, i=1,2, \ldots$ are a collection of minimum edge-to-vertex geodetic sets containing antipodal edges of $G$. Since each $S_{i}$ contains antipodal edges, we observe that every minimum edge-to-vertex geodetic set is independent of others. Therefore, each singleton set is the minimum forcing subset for exactly one minimum edge-to-vertex geodetic set of $G$. Hence, by Theorem $1.3(a)$ and $(b)$, we get $f_{e v}(G)=1$. Also, from Theorem $1.4(a)$ and $(b), f^{+}{ }_{e v}\left(S_{i}\right)=1$ for all $i=1,2, \ldots$ So that $f^{+}{ }_{e v}(G)=1$. Thus $f_{e v}(G)=1=f^{+}{ }_{e v}(G)$.

The following theorem is the interpretation of the previous theorem.

Theorem 2.7. Let $G$ be a connected graph with at least two $g_{e v}$-sets. If pairwise intersection of distinct minimum edge-to-vertex geodetic sets of $G$ is empty, then $f_{e v}(G)=1$ and $f^{+}{ }_{e v}(G)=1$.

Proof. Given that $G$ has at least two minimum $g_{e v}$-sets and for every minimum edge-to-vertex geodetic set $S_{i}, i=1,2, \ldots$ such that $S_{i} \cap S_{j}=\emptyset$. Therefore $S_{i}$ has an edge $u v$ such that $u v \notin S_{j}$ for every minimum edge-to-vertex geodetic $S_{j}$ different from $S_{i}$. Hence we obtain $f_{e v}(G)=1$. Since this is true for all minimum edge-to-vertex geodetic sets, we get $f^{+}{ }_{e v}(G)=1$.

The following table shows that the lower and upper forcing edge-to-vertex geodetic numbers of some wheel graphs.

Table 1

\begin{tabular}{|c|c|c|}
\hline Graph & $f_{e v}(G)$ & $f^{+}{ }_{e v}(G)$ \\
\hline$W_{3}$ & 1 & 1 \\
\hline$W_{4}$ & 2 & 2 \\
\hline$W_{5}$ & 1 & 1 \\
\hline$W_{6}$ & 1 & 1 \\
\hline$W_{7}$ & 2 & 3 \\
\hline$W_{8}$ & 2 & 2 \\
\hline$W_{9}$ & 1 & 1 \\
\hline$W_{10}$ & 2 & 3 \\
\hline
\end{tabular}


Corollary 2.8. For a wheel graph, $f_{e v}^{+}\left(W_{3 p}\right)=f_{e v}\left(W_{3 p}\right)=1$ if $p>1$

Proof. Let the vertices of $W_{3 p}(p>1)$ be $\left\{v_{1}, v_{2}, v_{3}, \ldots, v_{3 p+1}\right\}$. Note that the minimum edge-to-vertex geodetic sets of $W_{3 p}$ are $S_{1}=\left\{v_{1} v_{2}, v_{4} v_{5}, \ldots, v_{3 p-2} v_{3 p-1}\right\}, S_{2}=\left\{v_{2} v_{3}, v_{5} v_{6}, \ldots, v_{3 p-1} v_{3 p}\right\}, S_{3}=\left\{v_{3} v_{4}, v_{6} v_{7}, \ldots\right.$ $\left.v_{3 p} v_{1}\right\}$. It is clear that $S_{1} \cap S_{2}=S_{2} \cap S_{3}=S_{1} \cap S_{3}=\emptyset$. That is, pair wise intersection of minimum edge-tovertex geodetic sets of $W_{3 p}$ is empty. Hence by theorem 2.7, we have $f_{e v}\left(W_{3 p}\right)=1$. Since this is true for all minimum edge-to-vertex geodetic sets of $W_{3 p}$, we get $f^{+}{ }_{e v}\left(W_{3 p}\right)=1$.

Theorem 2.9. For a triangular snake, $G=T S_{n}$ of path $P_{n}(n>2), f_{e v}(G)=f^{+}{ }_{e v}(G)=1$.

Proof. Let $G=T S_{n}$ be a triangular snake obtained from the path $P_{n}$. Consider the vertices of $T S_{n}$ are $\left\{v_{1}, v_{2}, v_{3}, \ldots, v_{n}, u_{1}, u_{2}, u_{3}, \ldots, u_{n-1}\right\}$. The graph $G=T S_{n}$ is shown in Figure 1 .We can easily observe that $S_{1}=\left\{u_{1} v_{1}, u_{2} v_{3}, u_{3} v_{4}, \ldots, u_{n-1} v_{n}\right\}$ and $S_{2}=\left\{u_{1} v_{1}, u_{2} v_{2}, u_{3} v_{3}, \ldots, u_{n-2} v_{n-2}, u_{n-1} v_{n}\right\}$ are the only two minimum $g_{e v}$-sets of $G$, and some singleton sets are minimum forcing subsets for exactly one minimum $g_{e v}$-set of $G$. Hence, by Theorem $1.3(a)$ and $(b)$, we get $f_{e v}(G)=1$. Also, by Theorem 1.4 (a) and $(b), f^{+}{ }_{e v}\left(S_{i}\right)=1$ for all $i=1,2$. So that $f^{+}{ }_{e v}(G)=1$. Thus $f_{e v}(G)=1=f^{+}{ }_{e v}(G)$.

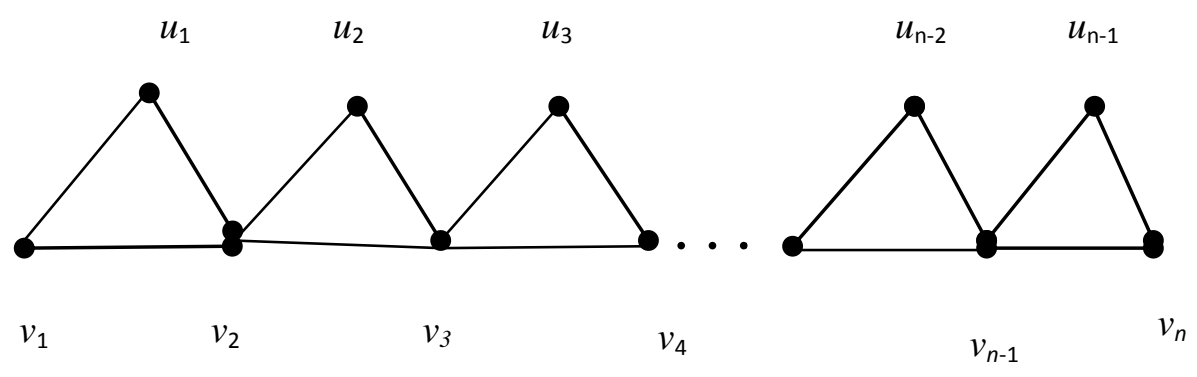

Figure 1.

Triangular snake $T S_{n}$ of path $P_{n}$

Theorem 2.10. For a square path $G=p_{n}{ }^{2}, f_{e v}(G)=f^{+}{ }_{e v}(G)=\left\{\begin{array}{l}0 \text { if } n \text { is even } \\ 2 \text { if } n \text { is odd }\end{array}\right.$.

Proof. Figure 2.

Let the vertices of the square path $p_{n}{ }^{2}$ be $\left\{v_{1}, v_{2}, v_{3}, \ldots, v_{n}\right\}$. The graph $G=p_{n}{ }^{2}$ is shown in

\section{Case (i): $n$ even $\& \boldsymbol{n}>2$.}

It is clear from the vertices of the square path $p_{n}^{2}, S=\left\{v_{1} v_{2}, v_{n-1} v_{n}\right\}$ is a unique minimum edgeto-vertex geodetic set of $G$. Since a square path, $1 \leq d(u, v) \leq 2$ for all $u, v$ in $G$, we have every vertices of $p_{n}{ }^{2}$ is either incident or lies on a geodesic joining of $v_{1} v_{2}$ and $v_{n-1} v_{n}$. Hence, by Theorem $1.3(a), f_{e v}$ $(G)=0$. Also, by Theorem $1.4(a), f^{+}{ }_{e v}(G)=0$. Thus $f_{e v}(G)=0=f^{+}{ }_{e v}(G)$.

\section{Case (ii): $n$ odd $\& \boldsymbol{n}>3$.}

Since $G$ has more than one minimum edge-to-vertex geodetic sets, and by Theorem 1.3 (a) $f_{e v}(G) \neq 0$ and by theorem 1.4(a) $f^{+}{ }_{e v}(G) \neq 0$. It is clear that, the sets $S_{1}=\left\{v_{1} v_{2}, v_{n-2} v_{n-1}, v_{n-1} v_{n}\right\}, S_{2}=$ $\left\{v_{1} v_{2}, v_{n-2} v_{n-1}, v_{n-2} v_{n}\right\}, S_{3}=\left\{v_{1} v_{3}, v_{2} v_{3}, v_{n-1} v_{n}\right\}, S_{4}=\left\{v_{1} v_{2}, v_{2} v_{3}, v_{n-1} v_{n}\right\}, S_{5}=\left\{v_{1} v_{2}, v_{n-2} v_{n}, v_{n-1} v_{n}\right\}, S_{6}=$ $\left\{v_{1} v_{2}, v_{1} v_{3}, v_{n-1} v_{n}\right\}$ are the only minimum edge-to-vertex geodetic sets of $p_{n}^{2}$. It is easily verified that, each singleton set is a subset of more than one minimum edge-to-vertex geodetic sets $S_{i}(1 \leq i \leq 6)$ and hence $f_{e v}(G) \neq 1$. Since $S_{3}$ is the unique minimum edge-to-vertex geodetic set containing $T=$ $\left\{v_{1} v_{3}, v_{2} v_{3}\right\}$, it follows that $f_{e v}\left(S_{3}\right)=2$. Hence $f_{e v}(G)=2$. But it is easily verified that the every two element subsets of $S_{i}$ are not contained in more than one minimum edge-to-vertex geodetic set $S_{i}(1 \leq i$ $\leq 6)$ so that $f_{e v}\left(S_{i}\right)=2$ for all $(1 \leq i \leq 6)$ and hence $f_{e v}^{+}(G)=2$. 


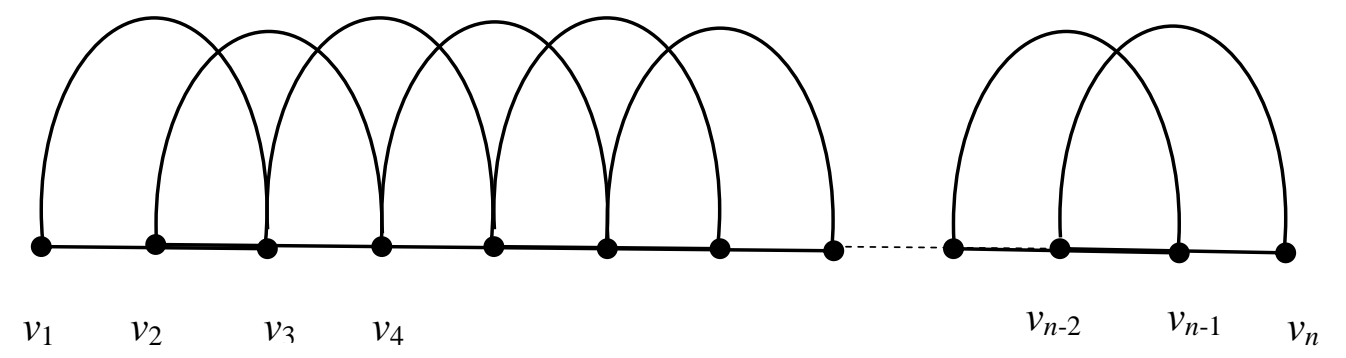

Figure 2.

Square path $p_{n}{ }^{2}$

\section{Conclusion}

In this paper we considered the lower and upper forcing edge-to-vertex geodetic numbers of some graphs. One can go for the general result on $f_{e v}(G)$ and $f^{+}{ }_{e v}(G)$, for every pair $a, b$ of integers with $0 \leq a \leq \mathrm{b} \leq \mathrm{c}$, there exists a connected graph $G$ such that $f_{e v}(G)=a, f^{+}{ }_{e v}(G)=\mathrm{b}$ and $g_{e v}(G)$ $=c$.

\section{ACKNOWLEDGEMENT}

The author of this article is supported by the University Grants Commission, New Delhi, through the minor research project for teachers (UGC XII- Plan) (F.NO:4-4/2014-15(MRPSEM/UGC-SERO))

\section{REFERENCES}

[1] Buckley F., Harary F., Distance in Graphs, Addition- Wesley, Redwood City, CA, 1990.

[2] Fu-Hsing Wang,Yue-li Wang, Jou-Ming Ghang, The lower and upper forcing geodetic numbers of block-cactus graphs, European Journal of Operational Research, 175(2006) 238-245.

[3] John J., Vijayan A. and Sujitha S., The forcing edge-to-vertex geodetic number of a graph, International Journal of Pure and Applied Mathematics (accepted).

[4] John J., Vijayan A. and Sujitha S., The forcing edge covering number of a graph, Journal of Discrete Mathematical Sciences \& Cryptography Vol.14 (2011), No.3, pp.249 - 259.

[5] Palani K., Nagarajan A., Forcing (G,D)-number of a Graph, International J.Math. Combin. Vol.3 (2011), 82-87.

[6] Rathod N.B., Kanan K.K. i, Some path related 4-cordial graphs, International Journal of Mathematics and Soft Computing, Vol.5, No.2 (2015), 21 - 27.

[7] Santhakumaran A.P., Center of graph with respect to edges, Scientia,19(2010) 13-23.

[8] Santhakumaran A.P. and John J., On the Edge-to-Vertex Geodetic Number of a Graph, Miskolc Mathematical Notes, 13(1)(2012) 107-119.

[9] Sujitha S., The upper forcing edge-to-vertex geodetic number of a graph, International Journal of Mathematics and Soft Computing, Vol.6, No.1 (2016), 29-38. 